\title{
How Structural Defects Affect the Mechanical and Electrical Properties of Single Molecular Wires
}

\author{
Matthias Koch, ${ }^{1, *}$ Zhi Li, ${ }^{2}$ Christophe Nacci, ${ }^{1,3}$ Takashi Kumagai, ${ }^{1}$ Ignacio Franco, ${ }^{2}$ and Leonhard Grill ${ }^{1,3}$ \\ ${ }^{1}$ Department of Physical Chemistry, Fritz. Haber Institute of the Max Planck Society, Faradayweg 4-6, 14195 Berlin, Germany \\ ${ }^{2}$ Department of Chemistry, University of Rochester, Rochester, New York 14627, USA \\ ${ }^{3}$ Department of Physical Chemistry, University of Graz, Heinrichstrasse 28, 8010 Graz, Austria
}

(Received 28 February 2018; revised manuscript received 25 April 2018; published 24 July 2018)

\begin{abstract}
We report how individual defects affect single graphene nanoribbons by scanning tunneling and atomic force microscopy pulling experiments simultaneously accessing their electrical and mechanical properties. The on-surface polymerization of the graphene nanoribbons is controlled by cooperative effects as theoretically suggested. Further, we find, with the help of atomistic simulations, that defects substantially vary the molecule-substrate coupling and drastically increase the flexibility of the graphene nanoribbons while keeping their desirable electronic properties intact.
\end{abstract}

DOI: 10.1103/PhysRevLett.121.047701

The supreme mechanical and electronic properties of graphene have attracted the attention of researchers from various fields [1-3]. Graphene nanoribbons (GNRs), onedimensional stripes of graphene, are expected to have similar properties [4]. Therefore, they are attractive candidates for molecular wires, a key component in molecular nanotechnology [5]. In addition to inheriting the electromechanical properties of graphene [1], GNRs offer a tunable band gap [6], which is crucial for electronics application. To study the tribological and electric properties of single molecular wires, various techniques have been developed to bridge the gap from macroscopic contacts to nanoscopic molecules, i.e., mechanical as well as scanning tunneling microscope break junction [7-10], scanning tunneling and atomic force microscopy (STM/AFM) pulling [11-17], and self-assembled monolayer (SAM) tunneling junctions [18].

In contrast to semiconductor devices, where crystal defects play a central role, in all studies on molecular wires, the molecules are considered as intact [8-10]. Computations show that even small chemical variations of the molecular structure influence their charge transport properties [19-21]. However, the role of structural defects in charge transport has been investigated experimentally for molecular wires not on the single-molecule level but only on a larger scale via their ordering and packing in SAMs [18]. In this Letter we study the electromechanical properties of randomly distributed structural defects with singlemolecule STM/AFM pulling experiments. This technique has the advantage that each molecular wire can be imaged before and after detaching it from the surface [16,22]. Therefore, the properties of individual defects can be probed by selecting individual molecular wires with specific (and exactly known) defect distributions. We investigate here how intrinsic defects influence the properties of single molecular wires. These defects do not result from a mixture of precursor molecules, where transitions between homogeneous sections of different composition occur, but truly a defect in the sense of an incomplete chemical structure. By using STM/AFM, we identify not only the presence but also the precise location of defects within an individual GNR, correlating its mechanical properties and conductance with its chemical structure.

Armchair GNRs are on-surface polymerized [23] from dibromo-dianthracene (DBDA) precursor molecules on $\mathrm{Au}(111)$ [24]. Experiments were performed with a commercial SPM from Omicron and an STM from Createc, operating at 5 and $10 \mathrm{~K}$, respectively. These molecular building blocks polymerize to polyanthracene at about $170^{\circ} \mathrm{C}$ and form flat GNRs by cyclodehydrogenation at higher temperatures $\left(400^{\circ} \mathrm{C}\right)$. Structural defects remain in the GNRs if insufficient temperatures of $330^{\circ} \mathrm{C}$ are used [Fig. 1(a)] [25]. To reduce steric hindrance between hydrogen atoms, every second not fully dehydrogenated anthryl group points away from the surface $[25,26]$ and appears in STM images as a protrusion [Fig. 1(b)] - a defect embedded in the GNR.

To control the defect density, we have varied the annealing temperature and find that the ribbon consists of defects only, i.e., pure polyanthracene, at $170{ }^{\circ} \mathrm{C}$ [Fig. 1(c)]. This number decreases only slowly up to $225^{\circ} \mathrm{C}$ where the major part of the molecule (90\%) is still not cyclodehydrogenated, but drops quickly to $10 \%$ at $280^{\circ} \mathrm{C}$. At intermediate temperatures we find that most defects, defined as one planar [marked orange in Fig. 1(a)] and one upward-pointing anthryl end (blue), appear in rows and isolated defects are rarely found, in agreement with previous observations [25]. From a statistical analysis it is very unlikely to find defects in a row and this probability decays quickly with the ribbon length and defect density [Fig. 1(d)]. This is in stark contrast to the experimentally 

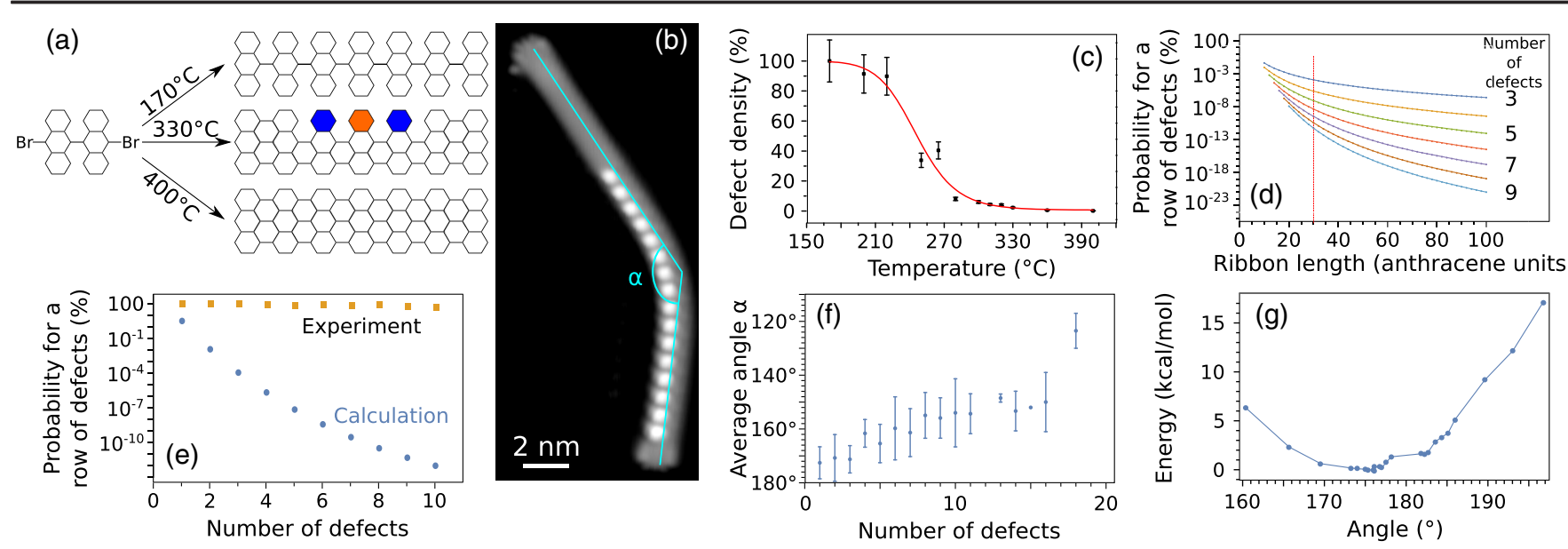

FIG. 1. (a) Illustration of the polymerization process on $\mathrm{Au}(111)$. Top: DBDA polymerizes at $170{ }^{\circ} \mathrm{C}$ to anthracene oligomers. Center: Not fully dehydrogenated ribbons are formed at $330^{\circ} \mathrm{C}$ as exemplified by the chemical structure of a GNR with two defects (upwardpointing anthryl groups indicated in blue, planar anthracene unit indicated in orange). Bottom: At $400^{\circ} \mathrm{C}$ fully dehydrogenated GNRs are formed. (b) STM image of a GNR adsorbed on $\mathrm{Au}(111)$ with several defects. (c) Average number of defects as a function of the annealing temperature. (d) Probability of finding a row of randomly distributed defects as a function of the ribbon length. Vertical line indicates the average length observed in experiments (13 nm and 15 dianthracene units) (e) Experimental distribution (squares) and calculated probability (circles) for a row of randomly distributed defects as a function of the number of defects. We calculated the probability for a ribbon which corresponds to the average experimental length (Supplemental Material [27]). (f) The average bending angle $\alpha$, as indicated in (b), as a function of the number of defects (Supplemental Material [28]). (g) Calculated energy of an adsorbed ribbon for different bending angles $\alpha$ (Supplemental Material [27]).

determined probability, which barely decreases with the defect density [Fig. 1(e)], confirming a nonrandom origin, in particular a cooperative mechanism as suggested by theory $[25,26]$.

Although a row of defects lowers the stiffness of the molecular backbone, the majority of the defected ribbons adsorb straight on the surface. Therefore, we quantify the influence of the defects on the flexibility of a GNR by measuring its curvature after a pulling experiment [Fig. 1(b)]. Despite the relatively large error, resulting from ribbons that readsorbed nearly straight, it is visible that the flexibility of adsorbed nanoribbons increases with the number of defects in the molecule [Fig. 1(f)]. This is in good agreement with our simulations. We calculated the energy for a ribbon adsorbed on the surface and we found that bending the ribbon towards the defected side of the molecule is energetically favored [Fig. $1(\mathrm{~g})]$. The reason for this behavior is the steric interaction between the hydrogen atoms and between the hydrogen and carbon atoms which increases for $\alpha>180^{\circ}$ (Supplemental Material [27]).

To study the influence of the defects on the mechanical properties, we performed AFM pulling experiments $[14,29]$. By applying a small amplitude $(\sim 80 \mathrm{pm})$ to the cantilever such that the effective stiffness is constant during one oscillation cycle, the frequency shift $\Delta f$ is proportional to the gradient of the vertical force acting in the pulling direction (i.e., perpendicular to the surface) [30]. The $Q$ factor is $\sim 25000$ for the AFM measurements and the resonance frequency is $22 \mathrm{kHz}$, when the tip is fully retracted. We find that $|\Delta f|$ does not decay as a function of the tip-sample distance [Fig. 2(a)], indicating that the sliding motion of the molecular portion adsorbed on the surface contributes only weakly to the signal [14]. To isolate the contribution of one individual defect, we have measured at large tip-sample distances and a tip height
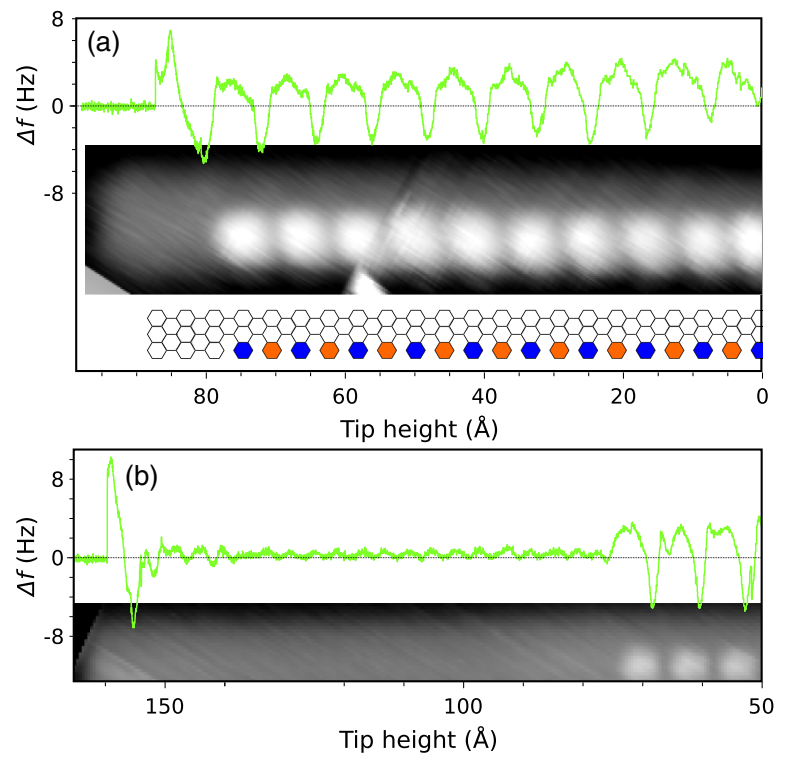

FIG. 2. [(a), (b)] $\Delta f$ traces with defects as shown in the STM image. The tip height of $0 \AA$ corresponds to a set point of $I=$ $100 \mathrm{pA}$ and $U=1 \mathrm{~V}$, and $I=100 \mathrm{pA}$ and $U=-1 \mathrm{~V}$ for (a) and (b), respectively. The bias voltage during the pulling experiment is 0 and $0.8 \mathrm{~V}$ for (a) and (b), respectively. 
of $0 \AA$ corresponds to a tip-sample distance of $\sim 6 \AA$. At these large tip-sample distances the influence from longrange as well as short-range interactions between tip and substrate is vanishing and the dominant contribution comes from the detachment process itself [31]. Instead, the $\Delta f$ signal oscillates with reproducible features around zero [Fig. 2(a)]. By comparing this signal with that of a nanoribbon that consists of two segments - one with and one without defects - the origin of the $\Delta f$ features becomes clear [Fig. 2(b)]: Each periodic feature corresponds to one defect-one planar and one upward-pointing unit-as evident from the comparison of the frequency shift curves with STM images [Figs. 2(a) and 2(b)]. By contrast, when the defect-free GNR segment is pulled upwards [tip heights above $80 \AA$ in Fig. 2(b)], only weak oscillations are observed, which we tentatively assign to the periodicity of the substrate, detected via the molecule-surface interaction at the surface-adsorbed nanoribbon terminus [14].

The average distance between two adjacent features in $\Delta f$ is $7.9 \pm 1.0 \AA$, in good agreement with the width of one dianthracene unit $(8.4 \pm 0.4 \AA$ from STM images). Note, that the free-standing part of the ribbon is slightly longer than the tip-surface distance, due to the curvature of the ribbon (Supplemental Movie [32]) [12,17]. Hence, the features in $\Delta f$ directly correlate with the defects within the ribbon, in terms of both number and position. Moreover, they appear precisely at the moment when the defected segment of the nanoribbon is detached from the surface and thus reflect the molecule-surface and intramolecular forces that are active in this process. Note, that the phase, excitation, and amplitude do not show any characteristic changes when a defect is lifted from the surface (Supplemental Material [28]). At the end of the pulling process, an intense negative $\Delta f$ peak appears [Figs. 2(a) and 2(b)], corresponding to a strong attractive force towards the surface that appears when the very end of the upright standing ribbon leaves the surface (Supplemental Movie [32]). Then the strong attractive force decreases, which is equivalent to a positive peak in $\Delta f$.

To develop atomistic insights into the experimental observations, we performed classical molecular dynamics simulations of the pulling [33,34]. The simulations were done in LAMMPS [35] using the OPLS force field (FF) for graphene [36], and a recently developed FF (vdW-FF) [33] that captures nonreactive metal-molecule interactions with the accuracy of high level electronic structure computations (specifically, DFT $+\mathrm{vdW}^{\text {surf }}$ ) [37]. We considered a GNR with 17 anthracene units on a fixed, flat $\mathrm{Au}(111)$ surface consisting of $(130 \times 130 \times 2)$ atoms. The surface $\left(36.3 \times 36.3 \mathrm{~nm}^{2}\right)$ is large compared to the molecule $(7.1 \mathrm{~nm})$, which ensures convergence of the adsorption energy with surface size. The starting structure is obtained via 300 to $4.7 \mathrm{~K}$ thermal annealing. Subsequently, the molecule is pulled from one terminal carbon with a constant speed of $v=10^{-6} \AA / \mathrm{fs}$ at $4.7 \mathrm{~K}$ using a Langevin thermostat. To mimic the elasticity of the gold tip, we use a $k=8 \mathrm{~N} / \mathrm{m}$ spring constant for the pulling device in silico along the pulling coordinate $z$ [38] and rigid perpendicular to $z$. The force exerted $F$ is measured via the deflection $\Delta$ of the pulling device from its equilibrium position $L=L_{0}+v t, F=-k \Delta(t)$, with $L_{0}$ as the initial
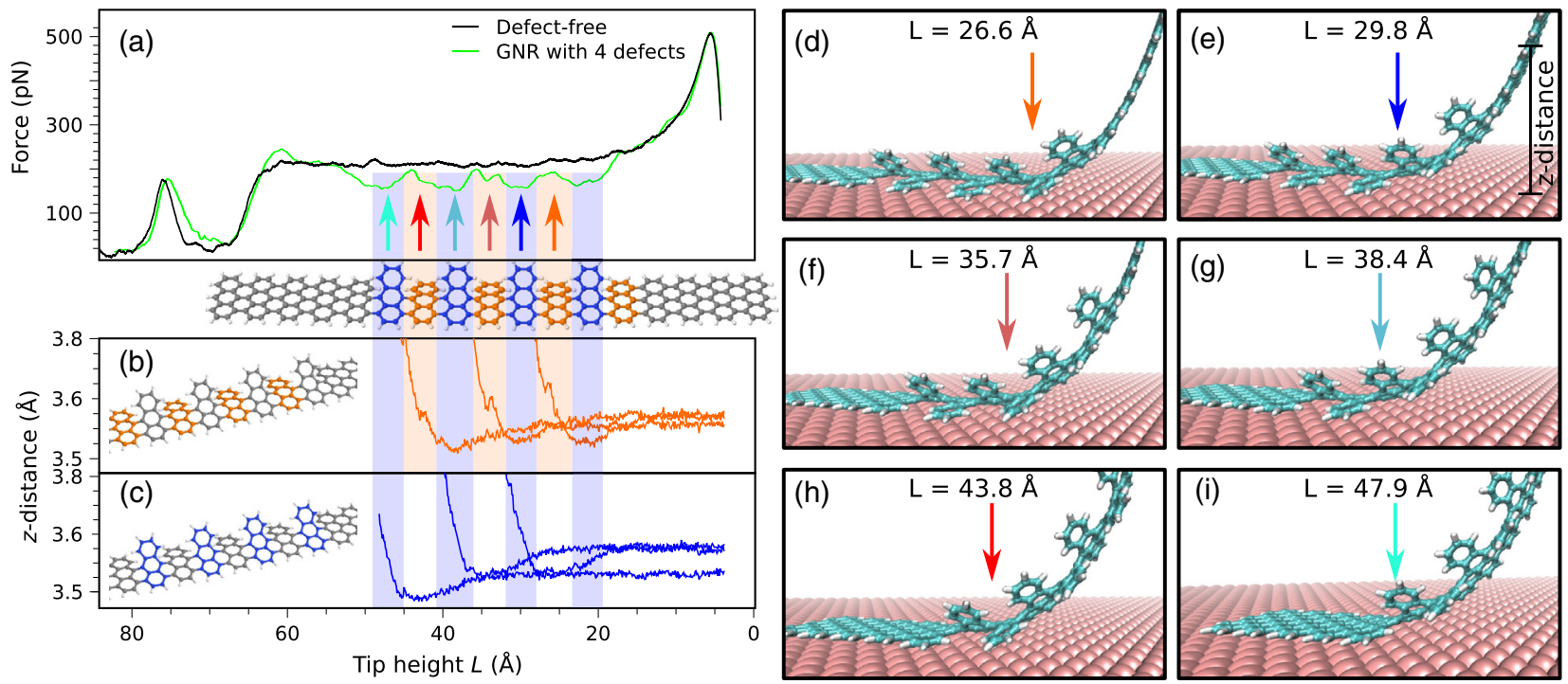

FIG. 3. (a) Simulated force for a pristine GNR (black) and a GNR with four defects (green). (b), (c) Height of the center of mass for the planar adsorbed (b) or the upward-pointing (c) benzene rings during pulling. For comparison, a scaled chemical structure of the defected GNR is plotted between (a) and (b). [(d)-(i)] Molecular snapshots associated with the force peaks and valleys during the pulling of the defected GNR. The planar or upward-pointing anthracene unit that is currently detached is indicated by the arrows in (d), (f), (h) and (e), (g), (i), respectively. 
position. To mimic experimental averaging over the STM tip oscillations, reported force curves are averages over $1.6 \AA$ in $L$.

Comparing the simulated force curves for two ribbons of identical length, but with only one of them containing defects [Figs. 3(a)-3(c)], the role of the defects becomes evident. As shown in Fig. 3(a), after an initial maximum (lifting the ribbon end from the substrate) a plateau of approximately constant force occurs for the pristine ribbon-in contrast to four distinct features that appear for the defected ribbon. When the first planar unit [Fig. 3(d)] is detached, its height (i.e., the $z$ coordinate of its center of mass) monotonically increases from 3.5 to 3.8 ̊̊ [Fig. 3(b)] and a force peak is observed in Fig. 3(a) (orange arrow at $L=26.6 \AA$ ). Next an upward-pointing anthracene unit [Fig. 3(e)] is lifted upwards as the $z$ coordinate of its center of mass starts to increase [Fig. 3(c)]. The weaker interaction of the upward-pointing anthracene unit with the surface results in a lower calculated force [blue arrow in Fig. 3(a) at $L=29.8 \AA$ ]. Therefore, the four valleys in the force trace arise from the weaker interaction of the upward-pointing benzene rings with the surface [blue regions in Figs. 3(a)-3(c) and Figs. 3(e), 3(g), and 3(i)]. By contrast, instantaneous force peaks appear when these unit have been detached from the surface [orange regions in Figs. 3(a)-3(d), 3(f), and 3(h)]. The periodicity of the force peaks is $\sim 8.5 \AA$, in agreement with the width of one dianthracene unit $(8.4 \AA)$. Before an anthracene unit is lifted off the surface the unit moves closer to the substrate to compensate the curvature of the ribbon before being detached (Supplemental Movie [32]).
To compare theory and experiment, we take into account that $\Delta f \propto-d F / d L$ and conclude that each negative $\Delta f$ peak (attractive region) in the measurements [Figs. 2(a) and 2(b)] reflects the detachment of a single anthracene unit that is adsorbed planar on the surface while the subsequent positive frequency (repulsive region) shift occurs when the neighboring upward-pointing anthracene group is lifted upwards [as sketched in Figs. 3(a)-3(c)].

When measuring the current through a single ribbon by STM pulling, the current shows a featureless decay as long as the intact part is lifted [Fig. 4(a)] [17]. Sudden current drops with a periodicity of $7.9 \pm 0.6 \AA$ appear when the tip height matches the positions of the four defects (I-IV) (see Fig. S2 [28] for more examples). When measuring current and $\Delta f$ simultaneously [Figs. 4(b) and 4(c)], the characteristic oscillations in both signals match with identical periodicity, confirming their assignment to the same individual defects. From comparison with our calculations [Fig. 3(a)], we can divide each feature in $\Delta f$ and current into two characteristic parts: (i) A negative peak in $\Delta f$ and decrease in current when the planar, stronger bound, unit is detached and (ii) a positive peak in $\Delta f$ and constant current when the weaker bound unit pointing up leaves the surface.

A possible reason for changes in the measured current could be a modification of the GNR electronic structure by the pulling process that deforms the ribbon. Our simulations show that the energy levels do not shift when an upward-pointing or planar anthracene is detached (computed as specified in Ref. [33]). However, when an upwardpointing anthracene is being lifted, the level broadening [Fig. 4(d)] in the transmission spectra is smaller, which indicates a weaker molecule-metal coupling and thus
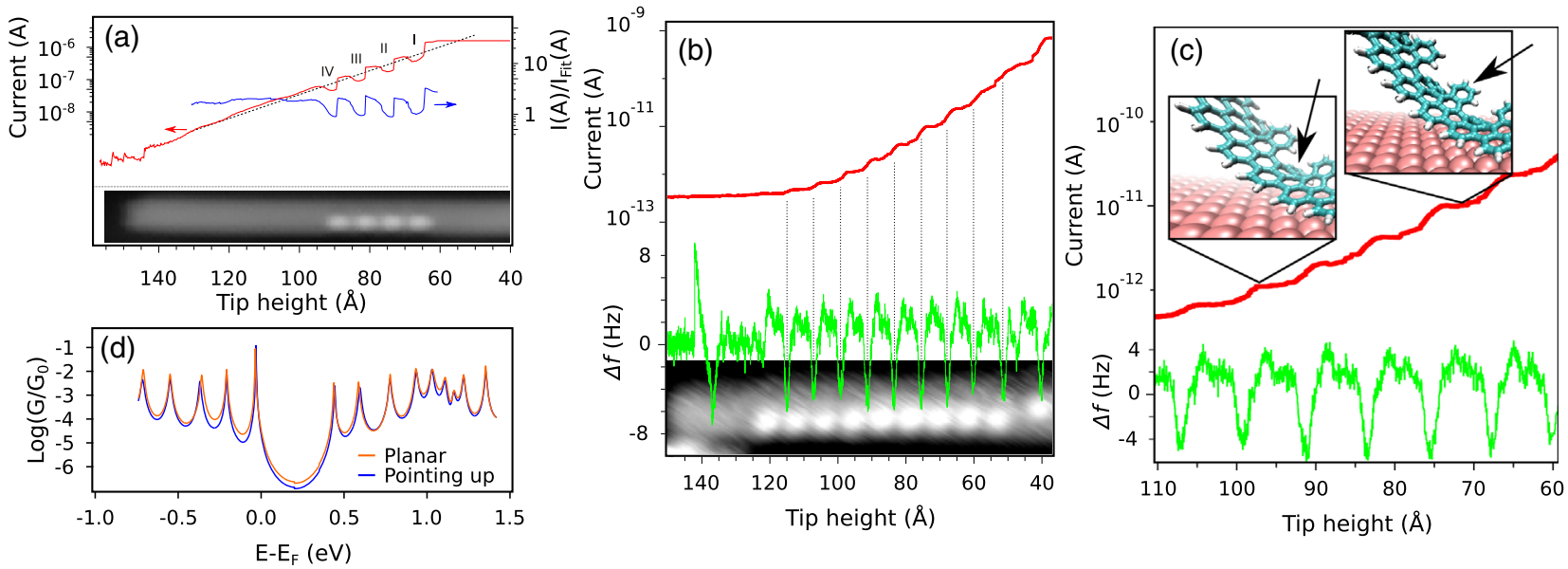

FIG. 4. (a) Pulling curve of a GNR with four defects at $+1.8 \mathrm{~V}$ bias voltage. The blue curve (current divided by its exponential fit) highlights the influence of the defects. (b) Pulling experiment where the current and the $\Delta f$ are recorded simultaneously. The bias voltage during the pulling experiment is $0.9 \mathrm{~V}$. The ribbon is fully detached at $\sim 140 \AA$ when the characteristic feature in the $\Delta f$ signal occurs. The corresponding drop in current is not visible due to the detection limit of the amplifier. The tip height of $0 \AA$ corresponds to a setpoint of $I=100 \mathrm{pA}$ and $U=0.1 \mathrm{~V}$, and $I=100 \mathrm{pA}$ and $U=0.9 \mathrm{~V}$ for (a) and (b), respectively. (c) Magnification of (b) with insets showing the detachment process. (d) Transmission curves of a GNR when an upward-pointing (blue) or a planar unit (orange) is detached. 
reduced charge transport of the molecule-surface contact (Supplemental Material [39]). Note, that the overall slope of the current is not affected by additional defects being detached from the surface [Figs. 4(a) and 4(b)]. Hence, the electrical conductance of a free-standing molecular wire is not lowered by the presence of defects. This is in agreement with our calculations, where the electronic states responsible for transport are similarly delocalized across the ribbon both in defected and pristine graphene. Hence, the defects do not break the conjugation of the molecule.

Our results reveal that structural defects substantially increase the mechanical flexibility of GNRs, but barely affect their overall electrical conductance. Introducing defects in two-dimensional materials is therefore of interest for future applications in nanotechnology where individual carbon-based molecular components are linked electrically and ensure at the same time enhanced flexibility in the architecture of the final circuits. In addition, these defects might also be used to locally tune the electronic or optical properties of the molecular wire [46], similar to semiconductor devices.

We thank Francisco Ample for substantial input about charge transport in molecular wires, Richard Pieber for help during the defect distribution experiments, and Martin Wolf for fruitful discussions and the support. T. K. acknowledges the support by the Deutsche Forschungsgemeinschaft through Sfb951.

*m.koch@fhi-berlin.mpg.de

[1] K. S. Novoselov, V. I. Falko, L. Colombo, P. R. Gellert, M. G. Schwab, and K. Kim, A roadmap for graphene, Nature (London) 490, 192 (2012).

[2] C. Lee, X. Wei, J. W. Kysar, and J. Hone, Measurement of the elastic properties and intrinsic strength of monolayer graphene, Science 321, 385 (2008).

[3] K. I. Bolotin, K. Sikes, Z. Jiang, M. Klima, G. Fudenberg, J. Hone, P. Kim, and H. Stormer, Ultrahigh electron mobility in suspended graphene, Solid State Commun. 146, 351 (2008).

[4] S. Dutta and S. K. Pati, Novel properties of graphene nanoribbons: a review, J. Mater. Chem. 20, 8207 (2010).

[5] L. A. Bumm, J. J. Arnold, M. T. Cygan, T. D. Dunbar, T. P. Burgin, L. Jones, D. L. Allara, J. M. Tour, and P. S. Weiss, Are single molecular wires conducting?, Science 271, 1705 (1996).

[6] M. Y. Han, B. Özyilmaz, Y. Zhang, and P. Kim, Energy Band-Gap Engineering of Graphene Nanoribbons, Phys. Rev. Lett. 98, 206805 (2007).

[7] A. Nitzan and M. A. Ratner, Electron transport in molecular wire junctions, Science 300, 1384 (2003).

[8] N. J. Tao, Electron transport in molecular junctions, Nat. Nanotechnol. 1, 173 (2006).
[9] S. V. Aradhya and L. Venkataraman, Single-molecule junctions beyond electronic transport, Nat. Nanotechnol. 8, 399 (2013).

[10] T. A. Su, M. Neupane, M. L. Steigerwald, L. Venkataraman, and C. Nuckolls, Chemical principles of single-molecule electronics, Nat. Rev. Mater. 1, 16002 (2016).

[11] R. Temirov, A. Lassise, F. B. Anders, and F. S. Tautz, Kondo effect by controlled cleavage of a single-molecule contact, Nanotechnology 19, 065401 (2008).

[12] L. Lafferentz, F. Ample, H. Yu, S. Hecht, C. Joachim, and L. Grill, Conductance of a single conjugated polymer as a continuous function of its length, Science 323, 1193 (2009).

[13] N. Fournier, C. Wagner, C. Weiss, R. Temirov, and F. S. Tautz, Force-controlled lifting of molecular wires, Phys. Rev. B 84, 035435 (2011).

[14] S. Kawai, M. Koch, E. Gnecco, A. Sadeghi, R. Pawlak, T. Glatzel, J. Schwarz, S. Goedecker, S. Hecht, A. Baratoff, L. Grill, and E. Meyer, Quantifying the atomic-level mechanics of single long physisorbed molecular chains, Proc. Natl. Acad. Sci. U.S.A. 111, 3968 (2014).

[15] G. Reecht, F. Scheurer, V. Speisser, Y. J. Dappe, F. Mathevet, and G. Schull, Electroluminescence of a Polythiophene Molecular Wire Suspended between a Metallic Surface and the Tip of a Scanning Tunneling Microscope, Phys. Rev. Lett. 112, 047403 (2014).

[16] C. Nacci, F. Ample, D. Bleger, S. Hecht, C. Joachim, and L. Grill, Conductance of a single flexible molecular wire composed of alternating donor and acceptor units, Nat. Commun. 6, 7397 (2015).

[17] M. Koch, F. Ample, C. Joachim, and L. Grill, Voltagedependent conductance of a single graphene nanoribbon, Nat. Nanotechnol. 7, 713 (2012).

[18] L. Jiang, L. Yuan, L. Cao, and C. A. Nijhuis, Controlling leakage currents: The role of the binding group and purity of the precursors for self-assembled monolayers in the performance of molecular diodes, J. Am. Chem. Soc. 136, 1982 (2014).

[19] T. C. Li and S.-P. Lu, Quantum conductance of graphene nanoribbons with edge defects, Phys. Rev. B 77, 085408 (2008).

[20] L. Chen, T. D. Huan, and R. Ramprasad, Electronic structure of polyethylene: Role of chemical, morphological and interfacial complexity, Sci. Rep. 7, 6128 (2017).

[21] F. C. Grozema, P. T. van Duijnen, Y. A. Berlin, M. A. Ratner, and L. D. A. Siebbeles, Intramolecular charge transport along isolated chains of conjugated polymers: Effect of torsional disorder and polymerization defects, J. Phys. Chem. B 106, 7791 (2002).

[22] P. H. Jacobse, A. Kimouche, T. Gebraad, M. M. Ervasti, J. M. Thijssen, P. Liljeroth, and I. Swart, Electronic components embedded in a single graphene nanoribbon, Nat. Commun. 8, 119 (2017).

[23] L. Grill, M. Dyer, L. Lafferentz, M. Persson, M. V. Peters, and S. Hecht, Nano-architectures by covalent assembly of molecular building blocks, Nat. Nanotechnol. 2, 687 (2007).

[24] J. Cai, P. Ruffieux, R. Jaafar, M. Bieri, T. Braun, S. Blankenburg, M. Muoth, A. P. Seitsonen, M. Saleh, X. Feng, K. Mullen, and R. Fasel, Atomically precise bottomup fabrication of graphene nanoribbons, Nature (London) 466, 470 (2010). 
[25] S. Blankenburg, J. Cai, P. Ruffieux, R. Jaafar, D. Passerone, X. Feng, K. Müllen, R. Fasel, and C. A. Pignedoli, Intraribbon heterojunction formation in ultranarrow graphene nanoribbons, ACS Nano 6, 2020 (2012).

[26] J. Björk, S. Stafström, and F. Hanke, Zipping up: Cooperativity drives the synthesis of graphene nanoribbons, J. Am. Chem. Soc. 133, 14884 (2011).

[27] See Supplemental Material at http://link.aps.org/ supplemental/10.1103/PhysRevLett.121.047701 for additional information on the calculation.

[28] See Supplemental Material at http://link.aps.org/ supplemental/10.1103/PhysRevLett.121.047701 for additional experimental data.

[29] S. Kawai, A. Benassi, E. Gnecco, H. Söde, R. Pawlak, X. Feng, K. Müllen, D. Passerone, C. A. Pignedoli, P. Ruffieux, R. Fasel, and E. Meyer, Superlubricity of graphene nanoribbons on gold surfaces, Science 351, 957 (2016).

[30] F. J. Giessibl, Advances in atomic force microscopy, Rev. Mod. Phys. 75, 949 (2003).

[31] S. Kawai, A. S. Foster, T. Björkman, S. Nowakowska, J. Björk, F. F. Canova, L. H. Gade, T. A. Jung, and E. Meyer, Van der Waals interactions and the limits of isolated atom models at interfaces, Nat. Commun. 7, 11559 (2016).

[32] See Supplemental Material at http://link.aps.org/ supplemental/10.1103/PhysRevLett.121.047701 for a movie of the molecular trajectory during pulling.

[33] Z. Li, A. Tkatchenko, and I. Franco, Modeling nonreactive molecule-surface systems on experimentally relevant time and length scales: Dynamics and conductance of polyfluorene on Au(111), J. Phys. Chem. Lett. 9, 1140 (2018).

[34] I. Franco, G. C. Schatz, and M. A. Ratner, Single-molecule pulling and the folding of donor-acceptor oligorotaxanes: Phenomenology and interpretation, J. Chem. Phys. 131, 124902 (2009).

[35] S. Plimpton, Fast parallel algorithms for short-range molecular dynamics, J. Comput. Phys. 117, 1 (1995).

[36] W. L. Jorgensen, D. S. Maxwell, and J. Tirado-Rives, Development and testing of the OPLS all-atom force field on conformational energetics and properties of organic liquids, J. Am. Chem. Soc. 118, 11225 (1996).
[37] V. G. Ruiz, W. Liu, E. Zojer, M. Scheffler, and A. Tkatchenko, Density-Functional Theory with Screened van der Waals Interactions for the Modeling of Hybrid Inorganic-Organic Systems, Phys. Rev. Lett. 108, 146103 (2012).

[38] H. Rascón-Ramos, J. M. Artés, Y. Li, and J. Hihath, Binding configurations and intramolecular strain in single-molecule devices, Nat. Mater. 14, 517 (2015).

[39] See Supplemental Material at http://link.aps.org/ supplemental/10.1103/PhysRevLett.121.047701 for additional information on the calculation, which includes Refs. [40-45].

[40] D. Benitez, N. D. Shapiro, E. Tkatchouk, Y. Wang, W. A. Goddard, and F. D. Toste, A bonding model for gold(I) carbene complexes, Nat. Chem. 1, 482 (2009).

[41] M. Tachibana, K. Yoshizawa, A. Ogawa, H. Fujimoto, and R. Hoffmann, Sulfur-Gold orbital interactions which determine the structure of Alkanethiolate/Au(111) selfassembled monolayer systems, J. Phys. Chem. B 106, 12727 (2002).

[42] M. D. Hanwell, D. E. Curtis, D. C. Lonie, T. Vandermeersch, E. Zurek, and G. R. Hutchison, Avogadro: an advanced semantic chemical editor, visualization, and analysis platform, J. Cheminform. 4, 17 (2012).

[43] R. Carey, L. Chen, B. Gu, and I. Franco, When can time-dependent currents be reproduced by the Landauer steady-state approximation?, J. Chem. Phys. 146, 174101 (2017).

[44] J. Hutcheson, I. Franco, N. Renaud, M. Carignano, M. A. Ratner, and G. C. Schatz, TRANSpull: Computes Pulling Coupled to Transport Properties of Single Molecules, DOI: 10.4231/D3ZS2KD79.

[45] S. R. Bahn and K. W. Jacobsen, An object-oriented scripting interface to a legacy electronic structure code, Comput. Sci. Eng. 4, 56 (2002).

[46] M. Chong, N. Afshar-Imani, F. Scheurer, C. Cardoso, A. Ferretti, D. Prezzi, and G. Schull, Bright electroluminescence from single graphene nanoribbon junctions, Nano Lett. 18, 175 (2018). 